\title{
Three-Phase Optimal Power Flow for Study of PV Plant Distributed Impact on Distribution Systems
}

\author{
Malinwo E. Ayikpa, Katia C. de Almeida and Guilherme C. Danielski \\ GSP-Labspot, Department of Electrical Engineering, Federal University of Santa Catarina 88040-900, Florianópolis, SC, Brazil
}

\begin{abstract}
This paper presents a TOPF (three-phase optimal power flow) model that represents photovoltaic systems. The PV plant is modeled in the TOPF as active and reactive power source. Reactive power can be generated or absorbed using the available capacity and the adjustable power factor of the inverter. The reduction of unbalance voltage and losses in the distribution systems is obtained by actions of reactive power control of the inverter. The TOPF is formulated by current balance equations and the PV systems are modeled via an equivalent circuit. The primal-dual interior point method is used to obtain the optimal operating points for the systems for different scenarios of solar irradiance and temperature, thus providing a detailed view of the impact of photovoltaic distributed generation.
\end{abstract}

Key words: Three-phase optimal power flow, photovoltaic generation, unbalance voltage, loss, primal-dual interior point method.

\section{Introduction}

The connection of generation sources to distribution systems can have significant impacts that need to be studied. Among the distributed generation sources, presenting the greatest growth is PV (photovoltaic). It is estimated that in 2023 there will be approximately 161 thousand PV generation systems in Brazil, which corresponds to the insertion of $835 \mathrm{MWp}$ into the grid [1]. The PV generation has intermittent features that are challenging for utilities. Unfortunately, there are still few computational tools to accurately assess the impact of distributed PV generation. One reason is the approximate representation of the PV generation and another is the use of single-phase equivalents for the distribution system. Tools based on single-phase equivalents may not provide the best operating solutions for three-phase systems, especially when circuits and/or loads are considerably unbalanced [2]. For these reasons, this work models the PV power plants in the TOPF (three-phase optimal power flow) from the PV module equivalent circuit and, with TOPF, analyzes the impact of the PV generation on the

Corresponding author: Malinwo Estone Ayikpa, electrical engineer, research field: electric power systems. distribution system.

The three-phase representation of the distribution system was first made in power flow programs that use the backward/forward sweeping method to obtain system voltages [3]. However, in Ref. [4] a three-phase current injection method was proposed, which, presented better convergence properties than the conventional method. The same formulation was used later in the three-phase optimal power flow [5].

Several papers on the TOPF problem have been published in recent years. Among them can be cited [6] which proposes a solution for an unbalanced FPO via the Quasi-Newton method; [7] which considers discrete control operations such as capacitor switching and taps adjustment of OLTCs; [8], based on semidefinite programming; [9] which extends the TOPF based on current injections for optimization of $n$-conductors systems and, [10] which achieves optimal adjustment of capacitor banks and voltage regulators to minimize active network loss. Some papers also present studies on the impact of distributed PV generation in the system $[5,11]$.

In studies on steady-state operation, PV plants are often represented by active power injections of values 
equal to their generation capacities. However, a more realistic view of the operating conditions of the system is obtained if the PV generation is calculated from measurements of solar irradiation and temperature. For this, the PV module can be represented by the five-parameter model $[12,13]$. This representation was used in the power flow problem [14] and, more recently, in the single-phase optimal power flow problem [15]. This paper describes a TOPF model based on the formulation proposed in Ref. [5], in which the PV plants are represented by the five-parameter model. The TOPF allows the injection of reactive power by the PV plant, which reduces the voltage unbalance and loss in the distribution systems.

The work is organized as follows: the next section explains how PV generation is calculated. Section 3 describes the TOPF problem used in the simulations. Section 4 analyzes the results obtained and, finally, Section 5 summarizes the main conclusions of the study.

\section{Photovoltaic Generation Calculation}

\subsection{The Five-Parameter Model}

The electrical power supplied by a photovoltaic module can be obtained from the equivalent electrical circuit of Fig. 1 [12]:

Applying Kirchhoff's 1st Law to the circuit, the current injected by the module is:

$$
I=I_{L}-I_{o}\left[\exp \left(\frac{V+I R_{s}}{V_{t} \hat{a}}\right)-1\right]-\frac{V+I R_{s}}{R_{s h}}
$$

where $I$ is the injected current, $V$ the terminal voltage, $I_{L}$ is the current generated by the incident light, $I_{0}$ is the saturation current of the diode, $V_{t}=$ $N_{s} k_{b} T_{c} / q$ is the thermal voltage of the array with $N_{s}$ cells connected in series, $k_{b}$ is the Boltzmann constant, $T_{c}$ is the cell temperature $(\mathrm{K}), q$ is the electron charge and $\hat{a}$ is the diode ideality constant. When one has the cells array, $R_{S}$ and $R_{s h}$ are the equivalent resistances.

Once the five-parameters $I_{L}, I_{0}, R_{S}, R_{S h}$ and $\hat{a}$, are known, the current, voltage and active power supplied by the module can be calculated in each scenario of temperature and solar irradiation. However, not all parameters are available, since manufacturers' datasheet of PV panels only provide the nominal open-circuit voltage, $V_{o c, n}$, the nominal short-circuit current, $I_{s c, n}$, voltage and current at the maximum power point, $V_{m p}$ and $I_{m p}$, the coefficients of temperature for open circuit voltage and short-circuit current, $K_{V}$ and $K_{I}$, and the maximum power produced experimentally, $P_{\max , e}$. The values are referred to STC (standard test conditions). Some manufacturers also provide I-V curves for different levels of radiation and temperature. In Ref. [13] an iterative method is proposed to obtain the equivalent circuit parameters from the information provided by the manufacturers. This method is used to calculate PV generation.

\subsection{Five-Parameter and DC Power Calculation}

The method is based on the fact that $I_{L}$ varies with the temperature and the incident solar irradiation in the panel according to:

$$
I_{L}=\left(I_{L, n}+K_{I} \Delta T\right) \frac{G}{G_{n}}
$$

where $I_{L, n}$ is the light-generated current at nominal conditions, $G$ the irradiation on the surface of the array, $G_{n}$ the nominal irradiation and $\Delta T=T-T_{n}$, where $T$ represents the measured temperature and $T_{n}$ the temperature at nominal conditions.

The saturation current of the diode is obtained in order to match the open circuit voltage with experimental data obtained for different temperatures. The starting point is the nominal saturation current

$$
I_{0, n}=\frac{I_{s c, n}}{\exp \left(V_{o c, n} / V_{t, n} \hat{a}\right)-1}
$$

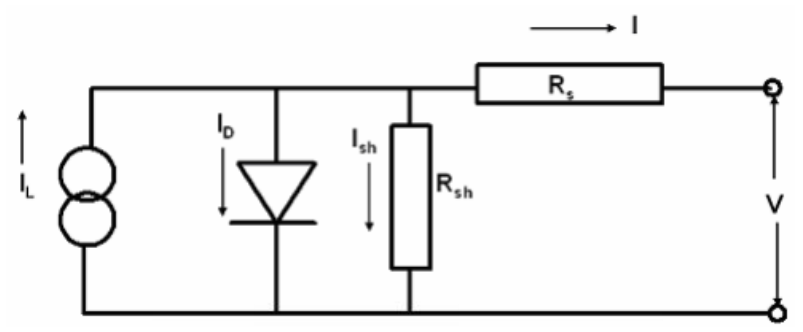

Fig. 1 Equivalent circuit for photovoltaic module. 
calculated on the thermal voltage of the $N_{S}$ cells connected in series, $V_{t, n}$, at the temperature $T_{n}$.

$I_{0}$ is obtained from Eq. (4) employing the voltage and current coefficients $K_{V}$ and $K_{I}$ and the temperature correction factor $\Delta T$ :

$$
I_{0}=\frac{I_{s c, n}+K_{I} \Delta T}{\exp \left(\frac{V_{o c, n}+K_{V} \Delta T}{V_{t, n} \widehat{a}}\right)-1}
$$

$\hat{a}$ is determined according to the type of technology used in the solar panel. For the module used (Hanwha polycrystalline silicon), $\widehat{a}=1,3$ [16].

$R_{S}$ and $R_{s h}$ are calculated from the assumption that only a pair $\left\{R_{s}, R_{s h}\right\}$ leads to the maximum power experimentally measured, $P_{\max , e}=V_{m p} I_{m p}$, in the conditions provided by the manufacturer. From Eq. (1), it follows that $R_{S}$ and $R_{s h}$ must respect:

$$
\begin{gathered}
P_{\max , e}=V_{m p}\left\{I_{L}-I_{0}\left[\exp \left(\frac{V_{m p}+R_{S} I_{m p}}{V_{t} \hat{a}}\right)-1\right]-\right. \\
\left.\frac{V_{m p}+R_{s} I_{m p}}{R_{S h}}\right\}
\end{gathered}
$$

To solve Eq. (5), $R_{S}$ is incremented from zero. $R_{s h}$ is initialized to the minimum value obtained by the inclination of the straight line segment joining the points on the I-V curve defined by the short-circuit and maximum power condition:

$$
R_{s h, \min }=\frac{V_{m p}}{I_{s c, n}-I_{m p}}-\frac{V_{o c, n}-V_{m p}}{I_{m p}}
$$

For every pair $\left\{R_{S}, R_{S h}\right\}$ the value of $I_{L}$ is updated using the relation between the currents of the circuit:

$$
I_{L, n}=\frac{R_{S}+R_{S h}}{R_{S}} I_{S c, n}
$$

Fig. 2 shows how $R_{S}$ and $R_{s h}$ are obtained.

Having found $R_{S}$ and $R_{S h}, I_{L}, I_{0}$ and the DC power generated $P_{\omega}$ can be calculated for every scenario of temperature and solar irradiation, according to the algorithm of Fig. 3.

\subsection{Inverter Modeling and AC Power Calculation}

The PV modules are connected in series forming strings and these are connected in parallel to the inverters. The number of modules per string, $N_{P V, S}$, and the number of strings, $N_{s t r}$, are chosen in order to achieve a good efficiency of DC-AC conversion. The

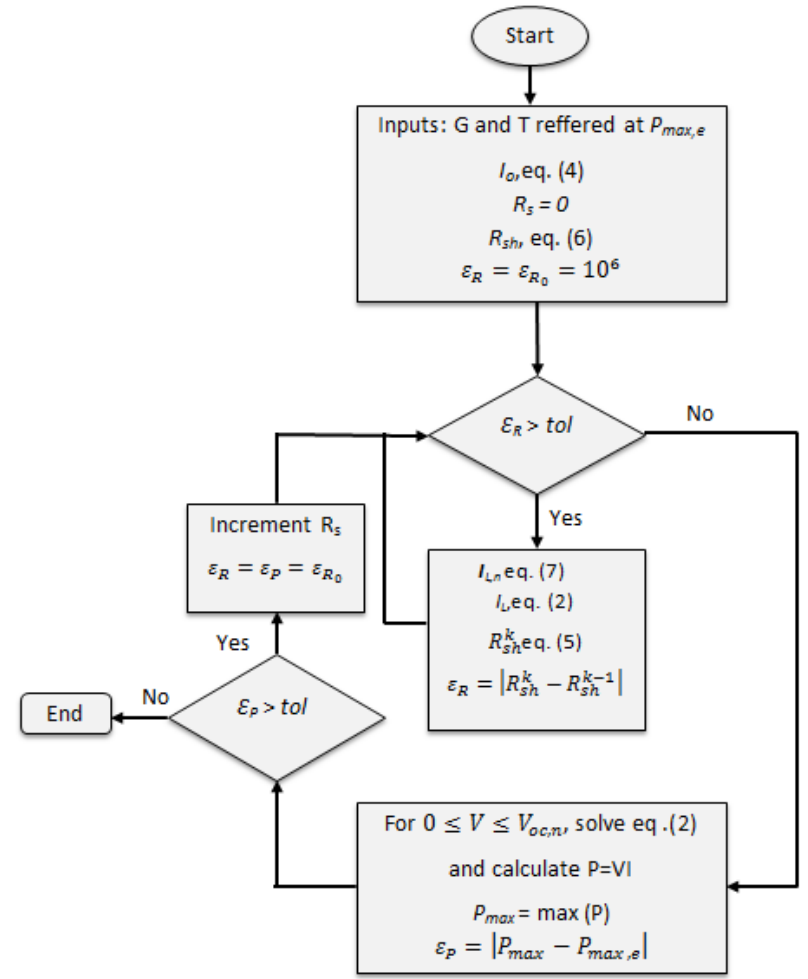

Fig. 2 Algorithm to determine $R_{s}$ and $R_{s h}$.

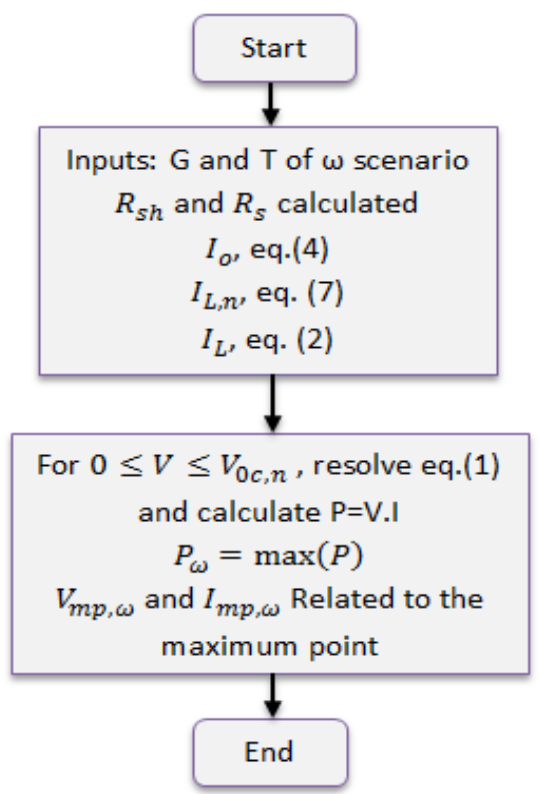

Fig. 3 Algorithm to determine $I_{o}, I_{L}$ and $P_{\omega}$.

inverter efficiency was calculated by means of exponential interpolation of the data indicated in the manufacturer's datasheet for each voltage curve, with $R^{2}=0.99$ as determination coefficient. The active $\mathrm{AC}$ power at the inverter output is:

$$
P_{P V, i n v, \omega}=\eta_{i n v, \omega} N_{P V, S} N_{s t r} P_{\omega}
$$


where the efficiency of the inverter is obtained by the function that interpolates the data of the manufacturer $\left(\eta_{i n v, \omega}=f_{V}\left(V_{m p, \omega}, I_{m p, \omega}\right)\right)$.

\section{Three-Phase Optimal Power Flow}

The TOPF problem is formulated from current injections [5]. In the problem, the active power supplied by the PV plants is not controllable. However, these power plants can generate reactive power since the inverters can operate with PF (power factors) less than 1 .

\subsection{Modeling of Photovoltaic Plant in the TOPF}

The photovoltaic power plant is connected to the grid via a transformer. Depending on the capacity of the photovoltaic plant, the connection can be single-phase or three-phase. The AC power supplied by the PV plant is:

$$
P_{P V, \omega}=P_{P V, i n v, \omega} N_{i n v}
$$

where $N_{i n v}$ is the number of inverters.

If the PV plant operates with adjustable power factor within the inverter limits, its reactive generation, $Q_{P V, \omega}$ must respect the nominal inverter capacity, $S_{i n v}^{\max }$, and its power factor, $P F_{i n v}$ :

$$
\left|Q_{P V}\right| \leq \min \left\{\sqrt{\left(\frac{P_{P V, \omega}}{P F_{i n v}}\right)^{2}-\left(P_{P V, \omega}\right)^{2}}, \sqrt{\left(S_{i n v}^{\max } \times N_{i n v}\right)^{2}-\left(P_{P V, \omega}\right)^{2}}\right\}
$$

\subsection{TOPF Problem Formulation and Its Resolution}

(A) Objective function. Two performance indices of the system were considered: minimum transmission losses,

$$
F_{1}=\min \sum_{k=1}^{n} \sum_{i=1}^{3}\left(P_{g_{k}}^{i}-P_{d_{k}}^{i}\right)
$$

where $n$ is the number of bus, $i$ the network phase index and $P_{g_{k}}^{i}$ and $P_{d_{k}}^{i}$ active power generation and load; and minimum voltage unbalance defined by the negative sequence component of all bus voltage system,

$$
F_{2}=\min \sum_{k=1}^{n}\left(V_{\mathrm{Re}, k,-}^{2}+V_{\mathrm{Im}, k-}^{2}\right)
$$

where,

$$
\begin{aligned}
& V_{k,-}=V_{k}^{a}+a^{2} V_{k}^{b}+a V_{k}^{c}, \quad a=1 \angle 120^{\circ} \\
& V_{k}^{a b c}=V_{\mathrm{Re}, k}^{a b c}+j V_{\mathrm{Im}, k}^{a b c}
\end{aligned}
$$

$V_{k}^{a b c}$ is the three-phase voltage phasor of the bus $k$ and the subscripts "Re" and "Im" indicate the real and imaginary part of the phasor.

(B) Equality constraints. The current balance in a given bus $k$ of the system is made by adding the current injections per phase of the elements connected to that bus:

$$
I_{g, k}^{a b c}-I_{d, k}^{a b c}-I_{k}^{a b c}=0
$$

where $I_{d, k}^{a b c}$ are the contributions of generators, $I_{d, k}^{a b c}$, the contributions of loads and $I_{k}^{a b c}$ the lines and transformers contributions connected to the phases $a, b$ and $c$ of this bus.

Separating the real and imaginary parts of Eq. (13), the current balance equations of TOPF are obtained:

$$
\begin{aligned}
& I_{\mathrm{Re}, g, k}^{a b c}-I_{\mathrm{Re}, d, k}^{a b c}-I_{\mathrm{Re}, k}^{a b c}=0 \\
& I_{\mathrm{Im}, g, k}^{a b c}-I_{\mathrm{Im}, d, k}^{a b c}-I_{\mathrm{Im}, k}^{a b c}=0
\end{aligned}
$$

The currents injected by the generator are expressed:

$$
I_{g, k}^{a b c}=I_{R e, g, k}^{a b c}+j I_{I m, g, k}^{a b c}=\left(\frac{P_{g, k}^{a b c}+j Q_{g, k}^{a b c}}{V_{k}^{a b c}}\right)^{*}
$$

where $P_{g_{k}}^{a b c}$ and $Q_{g_{k}}^{a b c}$ are active and reactive powers generated. On the other hand, the currents consumed by the loads are:

$$
I_{d, k}^{a b c}=I_{R e, d, k}^{a b c}+j I_{I m, d, k}^{a b c}=\left(\frac{P_{d, k}^{a b c}+j Q_{d, k}^{a b c}}{V_{k}^{a b c}}\right)^{*}
$$

where $P_{d_{k}}^{a b c}$ and $Q_{d_{k}}^{a b c}$ are active and reactive powers consumed.

The current contributions of the lines are calculated by the network equations, expressed in matrix form as:

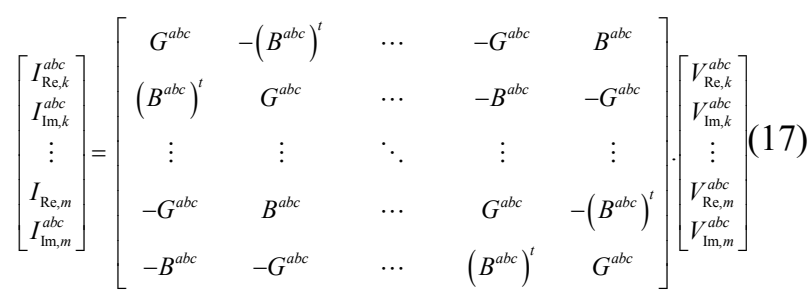

In Eq. (17), $G^{a b c}$ and $B^{a b c}$ are $3 \times 3$ matrix composed of the real and imaginary parts of the elements of the admittance matrix of the system, 
$I_{\mathrm{Re}, k}^{a b c}, I_{\mathrm{Re}, k}^{a b c}, I_{\mathrm{Im}, k}^{a b c}$ and $I_{\mathrm{Im}, m}^{a b c}$ are vectors $3 \times 1$ and $t$ indicates transposed matrix.

The transformers are considered fixed and represented by the $\pi$-equivalent circuit [17].

In order for the voltages in the reference bus (ref) to be delayed in $120^{\circ}$, the following equations are imposed:

$$
\begin{aligned}
& V_{\mathrm{Im}, \text { ref }}^{a}=0 \\
& V_{\mathrm{Im}, \text { ref }}^{b}-V_{\mathrm{Re}, \text { ref }}^{b} \tan (-2 \pi / 3)=0 \\
& V_{\mathrm{Im}, \text { ref }}^{c}-V_{\mathrm{Re}, \text { ref }}^{c} \tan (2 \pi / 3)=0
\end{aligned}
$$

Finally, in order to keep the voltage magnitude of the reference bus equal in the three-phases, the following equations can be inserted in the TOPF model:

$$
\begin{aligned}
& V_{\mathrm{Re}, r e f, a}^{2}+V_{\mathrm{Im}, \text { ref }, a}^{2}-V_{\mathrm{Re}, \text { ref }, b}^{2}-V_{\mathrm{Im}, r e f, b}^{2}=0 \\
& V_{\mathrm{Re}, r e f, a}^{2}+V_{\mathrm{Im}, r e f, a}^{2}-V_{\mathrm{Re}, r e f, c}^{2}-V_{\mathrm{Im}, r e f, c}^{2}=0
\end{aligned}
$$

(C) Inequality constraints. They represent operating limits and/or security aspects of the system. For each bus $k$ and phases $a, b$ and $c$, these constraints are:

$$
\begin{aligned}
& V_{\min , k}^{2 a b c} \leq V_{\mathrm{Re}, k}^{2 a b c}+V_{\mathrm{Im}, k}^{2 a b c} \leq V_{\max , k}^{2 a b c} \\
& P_{g_{\text {min, }, k}}^{a b c} \leq P_{g, k}^{a b c} \leq P_{g_{\text {max }, k}^{a b c}}^{a b c} \\
& Q_{g_{\min , k}}^{a b c} \leq Q_{g, k}^{a b c} \leq Q_{g_{\text {max }, k}}^{a b c}
\end{aligned}
$$

At the bus $k$, if there is a PV plant, in Eq. (20), $P_{g_{\text {min }, k}}^{a b c}=P_{g_{\text {max }, k}}^{a b c}=P_{P V, \omega}$ and $Q_{g_{k}}=Q_{P V, \omega}$ with limits given in Eq. (10).

The TOPF problem was solved by the primal-dual interior point method [18].

\section{Results}

The studies were performed with the IEEE34 bus system [19] and a 70-bus system (SIS70) [20]. Solar irradiation and temperature data were obtained at the INMET station in Santa Marta (SC) in January, April, July and October 2014. Data from the Hanwha SF220-30-1P240L (240Wp) panel and the SUNNY TRIPOWER 12000TL-US (12 kVA, FP $\geq 0.8$ ) inverter were used as well.

\subsection{Output Power Generation of the Inverter}

Fig. 4 shows the output power of the inverter on days considered taking into account SF220-30-1P240L panels. Such power is affected by the efficiency of the inverter, which varies from about $80 \%$ to about $97 \%$ depending on the irradiation and temperature. Thus, it changes over the days and also over the seasons.

\subsection{Impact of PV Generation on the System}

The PV plants were formed by connecting 48 SF220-30-1P240L panels per inverter, 24 of which were connected in series $\left(N_{P V, S}=24\right.$ and $\left.N_{s t r}=2\right)$. The number of inverters was chosen according to the capacity of the plant. The studies initially suppose two three-phase PV plants of the same capacity, connected in bus 890 and 848 of the IEEE34 example and in bus $58 \& 62$ of example SIS70.

(A) Results for a specific scenario of irradiation and temperature. The following results were obtained with the plants operating at maximum power on a summer day (02/01/2014). Table 1 indicates the TOPF solutions for the case without PV (base), with PV plants operating with a power factor unit or higher than 0.8 . The voltage unbalance base case is the state unbalance of the system with the solution of minimum losses. It is observed that the insertion of the power plants: (i) reduces the losses substantially, since the loads are supplied locally; and (ii) reduces the voltage unbalance.

Table 2 shows the power injected in the reference bus (substation) and the PV plants in cases of minimum

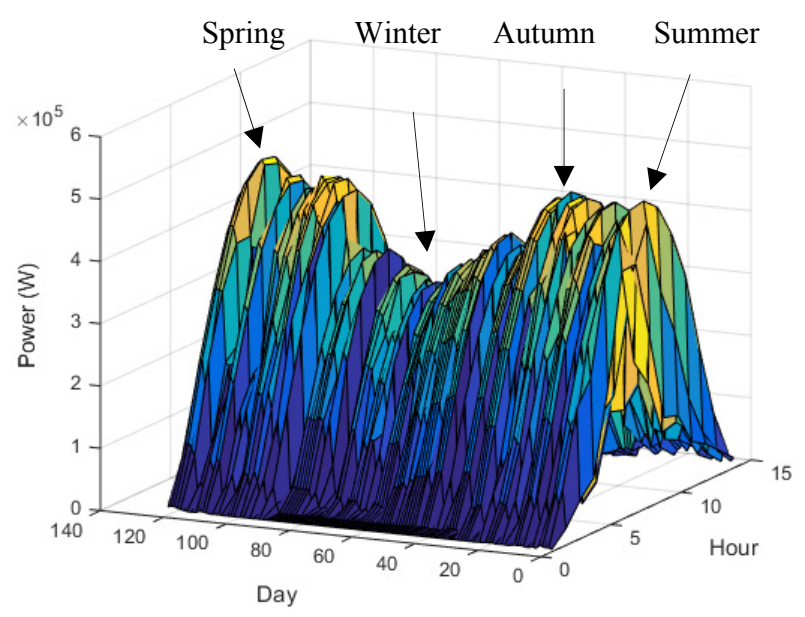

Fig. 4 Inverter output power. 
loss of the IEEE34 system. Note that in the base case, the substation supplies on average $623 \mathrm{~kW}$ and 127 kVAr per phase.

Table 2 shows that the active power injection drops to $283 \mathrm{~kW} /$ phase with the PV plants operating at the power factor unit level. Reactive injection drops to 43 $\mathrm{kVAr}$ /phase when PV plants operate with FP $\geq 0.8$. Note that the PV plants have very distinct participations in the control of reactive power of the systems.
Figs. 5 and 6 show the voltages profile of both systems. It is observed that the control of reactive power by PV plants makes it possible to operate with higher voltages and less unbalance, reducing the losses in the circuits.

Table 3 indicates the power injected by the substation and the PV plants of the SIS70 system in case where minimizing unbalance between phases. In the base case, the substation injects in the system in average $900 \mathrm{~kW}$ and $710 \mathrm{kVAr}$ per phase. With the

Table 1 Impact of PV plants on losses and voltage unbalance.

\begin{tabular}{lllllll}
\hline \multirow{2}{*}{ Sist. } & \multicolumn{3}{c}{ Min. losses $(\mathrm{kW})$} & \multicolumn{2}{c}{ Min. Unbal. (\%) } \\
\cline { 2 - 7 } & \multirow{2}{*}{ Base } & FV & FV & \multirow{2}{*}{ Base } & FV & FV \\
IEEE34 & 100.02 & 19.72 & 15.86 & 0.081 & 0.029 & 0.030 \\
SIS70 & 37.43 & 19.77 & 16.48 & 0.11 & 0.062 & 0.064 \\
\hline
\end{tabular}

Table 2 Power injections-Min. losses, IEEE34.

\begin{tabular}{|c|c|c|c|c|c|c|}
\hline \multirow{3}{*}{ Base case } & \multicolumn{6}{|c|}{ Active power $(\mathrm{kW})$ and reactive power (kVar) } \\
\hline & $\mathrm{Pg}_{\text {refa }}$ & $\mathrm{Pg}_{\text {refb }}$ & $\mathrm{Pg}_{\text {refc }}$ & $\mathrm{Qg}_{\text {refa }}$ & $\mathrm{Qg}_{\mathrm{refb}}$ & $\mathrm{Qg}_{\mathrm{refc}}$ \\
\hline & 640.02 & 615.46 & 613.54 & 138.01 & 119.94 & 122.36 \\
\hline \multirow{4}{*}{ PV Plant with/ $\mathrm{PF}=1$} & $P g_{\text {refa }}$ & $\mathrm{Pg}_{\mathrm{refb}}$ & $\mathrm{Pg}_{\text {refc }}$ & $\mathrm{Qg}_{\text {refa }}$ & $\mathrm{Qg}_{\mathrm{refb}}$ & $\mathrm{Qg}_{\mathrm{refc}}$ \\
\hline & 300.01 & 275.86 & 272.01 & 91.43 & 76.35 & 76.39 \\
\hline & $\mathrm{P}_{\mathrm{PVa}}$ & $\mathrm{P}_{\mathrm{PVb}}$ & $\mathrm{P}_{\mathrm{PV} c}$ & $\mathrm{Q}_{\mathrm{PVa}}$ & $\mathrm{Q}_{\mathrm{PVb}}$ & $\mathrm{QPVc}_{\mathrm{P}}$ \\
\hline & 314 & 314 & 314 & - & - & - \\
\hline \multirow{5}{*}{$\begin{array}{l}\text { PV Plant with/ PF } \geq \\
0.8\end{array}$} & $P g_{\text {refa }}$ & $P g_{\text {refb }}$ & $P g_{\text {refc }}$ & $\mathrm{Qg}_{\text {refa }}$ & $\mathrm{Qg}_{\mathrm{refb}}$ & $\mathrm{Qg}_{\text {refc }}$ \\
\hline & 298.77 & 274.65 & 270.70 & 53.31 & 38.52 & 38.50 \\
\hline & $\mathrm{P}_{\mathrm{PVa}}$ & $\mathrm{P}_{\mathrm{PVb}}$ & $\mathrm{P}_{\mathrm{PV} \mathrm{c}}$ & $\mathrm{Q}_{\mathrm{PVa}}$ & $\mathrm{Q}_{\mathrm{PVb}}$ & $\mathrm{Q}_{\mathrm{PVc}}$ \\
\hline & 157 & 157 & 157 & 70.73 & 70.73 & 70.73 \\
\hline & 157 & 157 & 157 & -34.15 & -34.15 & -34.15 \\
\hline
\end{tabular}
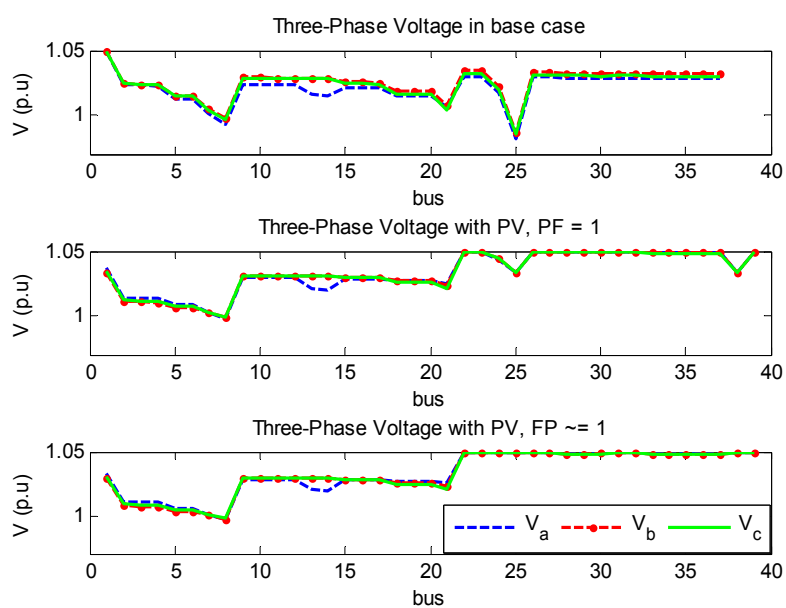

Fig. 5 Voltages, case of Min. losses-IEEE34.
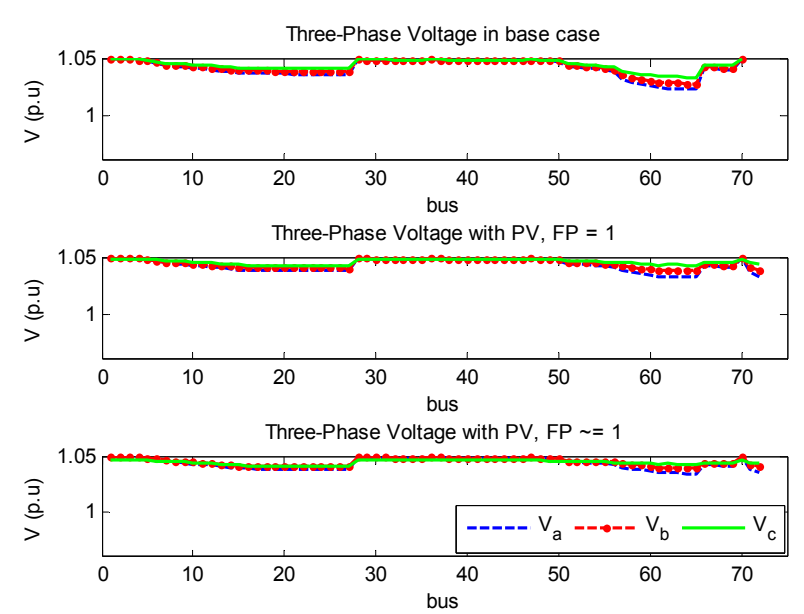

Fig. 6 Voltages, case of Min. losses-SIS70. 
Table 3 Power injections-Min. Unbal., SIS70.

\begin{tabular}{|c|c|c|c|c|c|c|}
\hline \multirow{3}{*}{ Base case } & \multicolumn{6}{|c|}{ Active power $(\mathrm{kW})$ and reactive power (kVar) } \\
\hline & $\mathrm{Pg}_{\text {refa }}$ & $\mathrm{Pg}_{\mathrm{refb}}$ & $P g_{\text {refc }}$ & $\mathrm{Qg}_{\mathrm{refa}}$ & $\mathrm{Qg}_{\mathrm{refb}}$ & $\mathrm{Qg}_{\mathrm{refc}}$ \\
\hline & $1,126.7$ & 898.3 & 671.4 & 889.50 & 710.21 & 531.63 \\
\hline \multirow{4}{*}{$\begin{array}{l}\text { PV Plant } \\
\text { with/ PF = } 1\end{array}$} & $\mathrm{Pg}_{\text {refa }}$ & $\mathrm{Pg}_{\text {refb }}$ & $\mathrm{Pg}_{\text {refc }}$ & $\mathrm{Qg}_{\mathrm{refa}}$ & $\mathrm{Qg}_{\mathrm{refb}}$ & $\mathrm{Qg}_{\mathrm{refc}}$ \\
\hline & 804.84 & 578.59 & 353.86 & 886.12 & 707.76 & 530.08 \\
\hline & $\mathrm{P}_{\mathrm{PVa}}$ & $\mathrm{P}_{\mathrm{PVb}}$ & $\mathrm{P}_{\mathrm{PV} c}$ & $\mathrm{QPVa}_{\mathrm{PV}}$ & $\mathrm{Q}_{\mathrm{PVb}}$ & $\mathrm{QPVc}_{\mathrm{P}}$ \\
\hline & 320 & 320 & 320 & - & - & - \\
\hline \multirow{5}{*}{ PV Plant with/ $\mathrm{PF} \geq 0.8$} & $P g_{\text {refa }}$ & $\mathrm{Pg}_{\text {refb }}$ & $\mathrm{Pg}_{\text {refc }}$ & $\mathrm{Qg}_{\mathrm{refa}}$ & $\mathrm{Qg}_{\text {refb }}$ & $\mathrm{Qg}_{\text {refc }}$ \\
\hline & 802.73 & 576.89 & 352.63 & 779 & 600.80 & 423.31 \\
\hline & $\mathrm{P}_{\mathrm{PVa}}$ & $\mathrm{P}_{\mathrm{PVb}}$ & $\mathrm{P}_{\mathrm{PV} \mathrm{c}}$ & $\mathrm{Q}_{\mathrm{PVa}}$ & $\mathrm{Q}_{\mathrm{PVb}}$ & $\mathrm{Q}_{\mathrm{PV} \mathrm{c}}$ \\
\hline & 157 & 157 & 157 & 47.21 & 47.21 & 47.21 \\
\hline & 157 & 157 & 157 & 59.04 & 59.04 & 59.04 \\
\hline
\end{tabular}

insertion of the PV plants, the active power injection in the substation drops to $579 \mathrm{~kW} /$ phase. Even when the plants operate with FP $\neq 1$, the injection of reactive is done mainly by the substation. Despite this, Fig. 7 indicates that if the PV plants provide reactive, the voltage profile becomes closer to the nominal. This aspect is also observed in Fig. 8 with PV plants injecting reactive power in the system IEEE34.

(B) Daily PV generation and its impact on the IEEE34 system. The operating solutions of minimum losses for the IEEE34 system are analyzed on a summer day (02/01/2014) and on a winter day (16/07/2014). The same scenario of irradiation and temperature was considered for the two PV plants, which implies that the plants generate the same active power.

Fig. 9 indicates the total active load of the system and the active powers injected by the substation and the PV plants on summer and winter days. The same load curve was considered in the two days. In the summer scenario, in the hours of higher solar irradiation and temperature, PV plants supply almost $50 \%$ of the system's load.

Fig. 10 shows the active power generation at each phase of each PV plant during summer day. The active power reaches the maximum $(157 \mathrm{~kW} /$ phase $)$ at $11 \mathrm{am}$. The average generation in the day is equal to 75 $\mathrm{kW} /$ phase, which equals $47 \%$ of the capacity of each PV plant.
Figs. 11 and 12 indicate reactive generation of PV plants on summer day. Substantial variations in this generation are observed, mainly in the PV plant 2 between $11 \mathrm{~h}$ and $12 \mathrm{~h}$ and between $14 \mathrm{~h}$ and $15 \mathrm{~h}$.
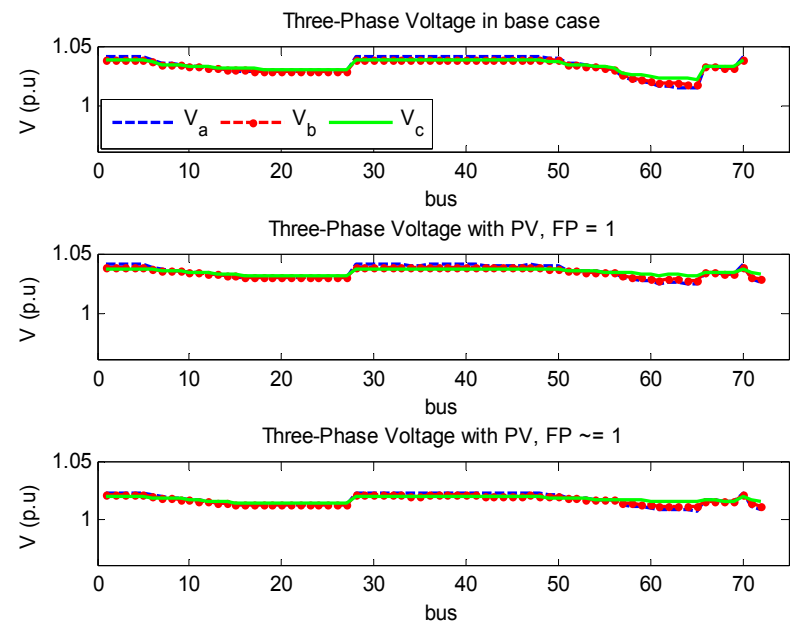

Fig. 7 Voltages, case of Min. Unbal.-SIS70.
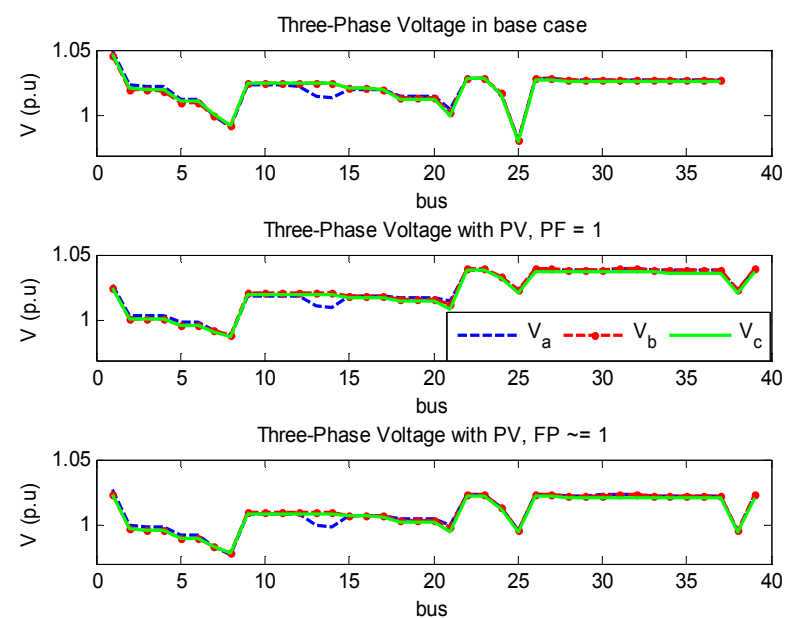

Fig. 8 Voltages, case of Min. Unbal. - IEEE34. 

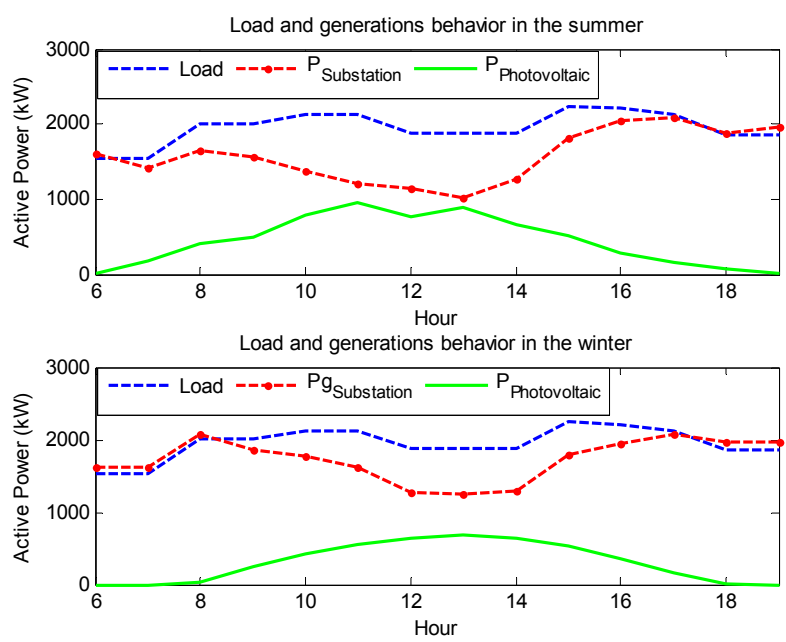

Fig. 9 Total load and generations of IEEE34 system.

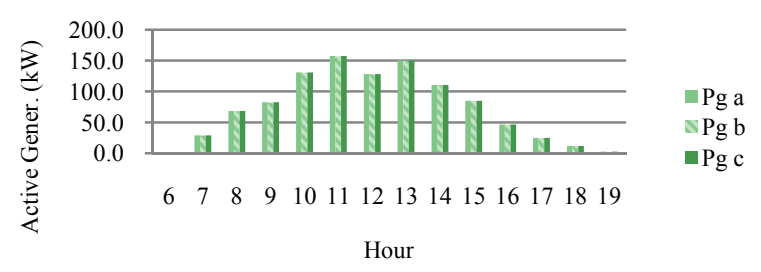

Fig. 10 Active generation—summer.

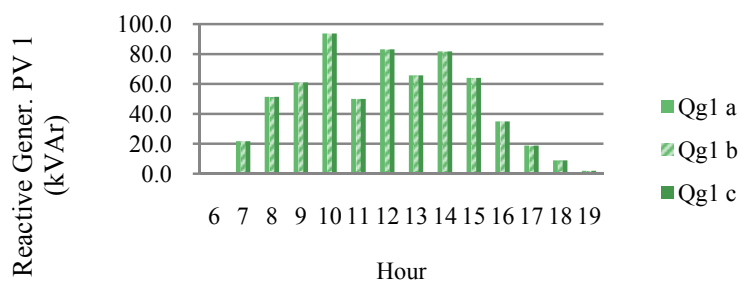

Fig. 11 Reactive generation of PV plant 1-summer, IEEE34.
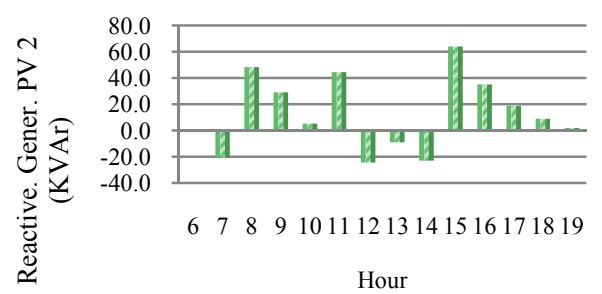

- Qg2 a

ag2 b

$\square \mathrm{Qg} 2 \mathrm{c}$

Hour

Fig. 12 Reactive generation of PV plant 2-summer, IEEE34.

In the winter scenario, the maximum active generation of each plant is $113 \mathrm{~kW} /$ phase and the average is $51 \mathrm{~kW} /$ phase or $72 \%$ and $32 \%$ of available capacity. The control of reactive power plants also decreases in this new scenario. However, since the winter load is equal to the summer load, the way PV plants participate in the voltage control remains the same, i.e., the central 1 injects reactive in the system, while the central 2, depending on the time of the day, injects or absorbs reactive system.

Finally, Fig. 13 indicates the behavior of the voltage in a critical bus of the system throughout the days of summer and winter. Note a variation of $7 \%$ in this voltage between the hours of maximum and minimum PV generation.

(C) Daily PV generation and its impact on the SIS70 system. The operating solutions of minimum unbalance for the SIS70 system are analyzed in this section with the same active power generated by both PV plants.

Fig. 14 indicates that PV plants supply almost 30\%
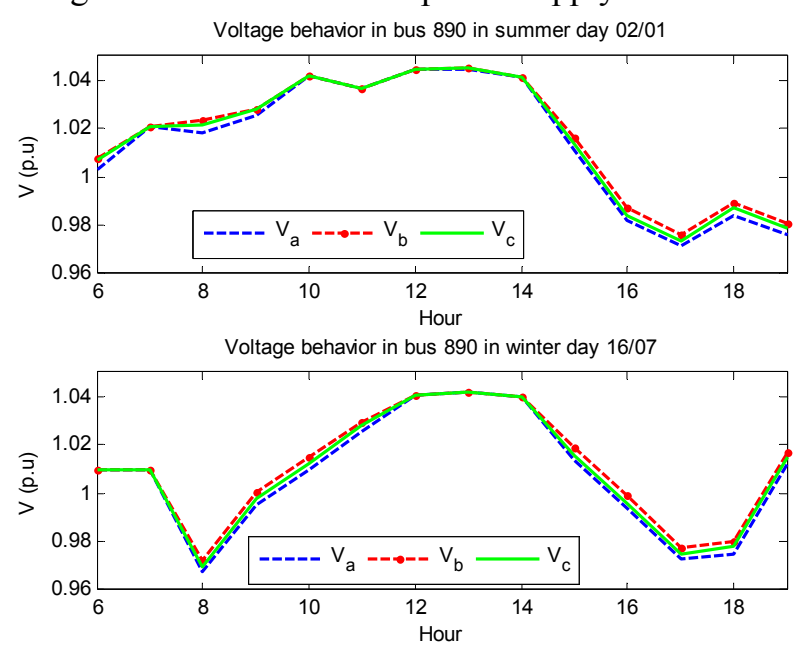

Fig. 13 Critical bus voltage behavior.
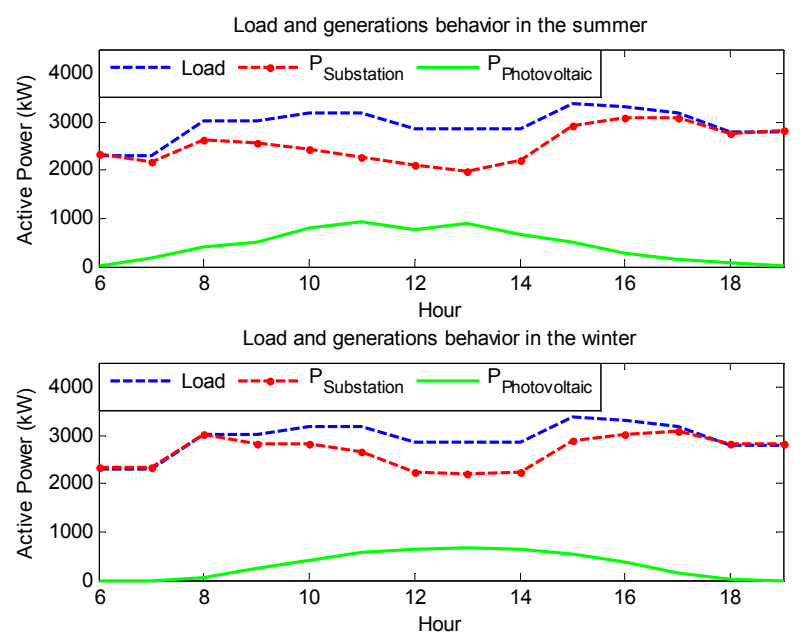

Fig. 14 Total load and generations of SIS70 system. 
of the system's load in the summer with its maximum generation. This generation is even smaller in the winter.

The reactive power generations of PV plants on summer day are shown in Figs. 15 and 16, where both plants inject reactive power to the grid. Note that PV plant 2 contributes to $67 \%$ of the reactive power control of both PV plants, thus having an important role in voltage control.

(D) Impact of single phase PV plants. Fig. 17 shows

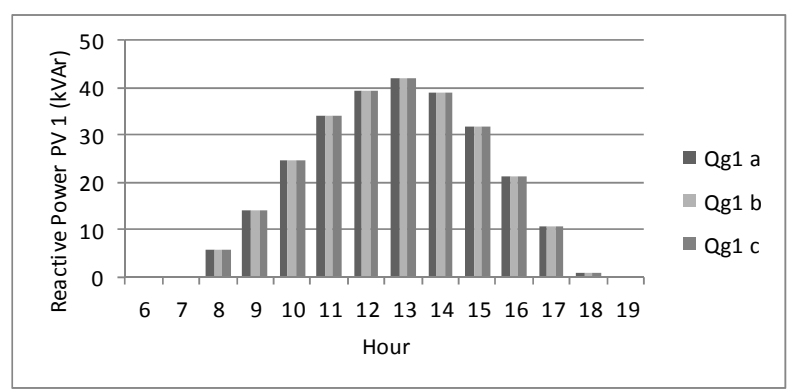

Fig. 15 Reactive generation of PV plant 1-summer, SIS70.

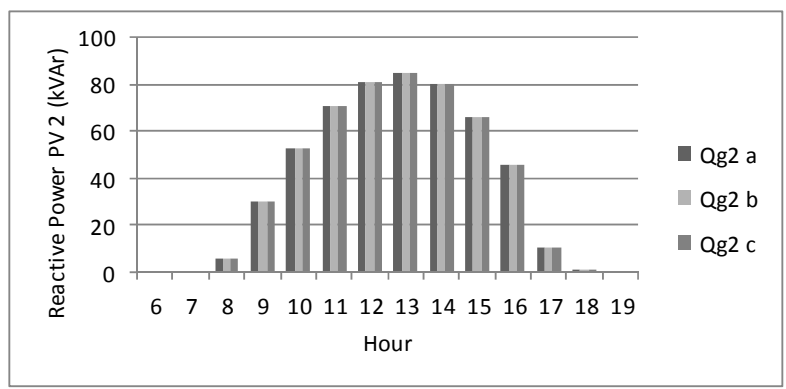

Fig. 16 Reactive generation of PV plant 2-summer, SIS70.
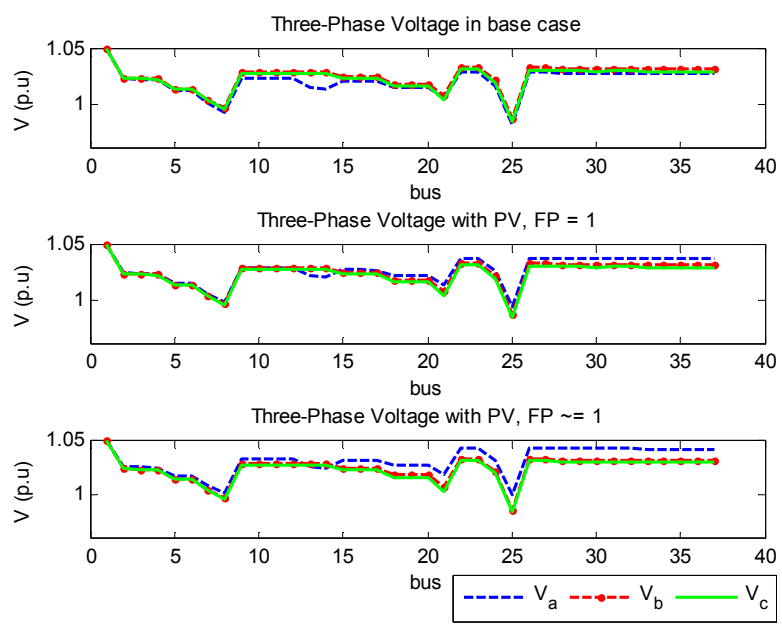

Fig. 17 Voltage, Min. loss-IEEE34, PV $1 \phi$ in Phase $a$. the behavior of IEEE34 system voltages in the minimum losses solutions when $10 \mathrm{PV}$ plants of $15 \mathrm{~kW}$ are connected in the phase $a$ of 10 bus of this system. It can be seen that such insertion significantly increases the voltages in phase $a$ and the voltage unbalance of the system. This aspect consolidates the importance of the three-phase representation of the power plants in the OPF (optimal power flow).

\section{Conclusion}

The use of the five-parameter equivalent circuit enables a very accurate representation of the PV generation in the TOPF problem and a detailed analysis of the impact of the distributed PV generation. The simulations confirm the variability of this generation and show the importance of PV plants in voltage control. The three-phase representation of the power plants in the OPF enables the voltage unbalance between the phases to be reduced. The methodology used to obtain the PV generation is still approximate and the TOPF program needs to be improved to represent control equipment of the distribution systems. The improvement of these tools is the next step of the research being developed.

\section{Acknowledgments}

The authors would like to thank CAPES and CNPq for their support in the development of this research.

\section{References}

[1] Empresa de Pesquisa Energética. 2014. "Inserção da geração fotovoltaica distribuída no Brasil - condicionantes e impactos." Brasilia.

[2] von Jouanne, A., and Banerjee, B. 2001. "Assessment of Voltage Unbalance." IEEE Trans. on Power Delivery, 782-90.

[3] Cheng, C. S., and Shirmohammadi, D. 1995. "A Three-Phase Power Flow Method for Real-Time Distribution System Analysis." IEEE Trans. on Power System 10 (2): 671-9.

[4] Garcia, P. A. N., Pereira, J. L. R., Carneiro, S., da Costa, V. M., and Martins, N. 2000. "Three-Phase Power Flow Calculations Using the Current Injection Method." IEEE Trans. on Power Systems 15 (2): 508-14. 
[5] Araujo, L. R., Penido, D. R. R., Carneiro, S., and Pereira, J. L. R. 2013. "A Three-Phase Optimal Power Flow Algorithm to Mitigate Voltage Unbalance." IEEE Trans. on Power Delivery 28 (4): 2394-402.

[6] Bruno, S., Lamonaca, S., Stecchi, U., and La Scala, M. 2011. "Unbalanced Three-Phase Optimal Power Flow for Smart Grids." IEEE Trans. on Industrial Electronics 58 (10): 4504-13.

[7] Paudyal, S., Cañizares, C. A., and Bhattacharya, K. 2011. "Optimal Operation of Distribution Feeders in Smart Grids.” IEEE Trans. on Industrial Electronics 58 (10): 4495-503.

[8] Dall'Anese, E., Zhu, H., and Giannakis, G. B. 2013. "Distributed Optimal Power Flow for Smart Microgrids." IEEE Trans. on Smart Grid 4 (3): 1464-75.

[9] de Araujo, L. R., Penido, D. R. R., and de Alcântara Vieira, F. 2013. "A Multiphase Optimal Power Flow Algorithm for Unbalanced Distribution Systems." Electrical Power and Energy Systems, Elsevier 53: 632-42.

[10] Baran, A. R., and Fernandes, T. S. P. 2016. "A Three-Phase Optimal Power flow Applied to the Planning of Unbalanced Distribution Networks." Electrical Power and Energy Systems, Elsevier 74: 301-9.

[11] Bozchalui, M. C., Jin, C. R., and Sharma, R. 2014. "Rolling Stochastic Optimization Based Operation of Distribution Systems with PVs and Energy Storages." In Innovative Smart Grid Technologies Conference (ISGT), 2014 IEEE PES, 1-5.

[12] De Soto, W., Klein, S. A., and Beckman, W. A. 2006.
"Improvement and Validation of a Model for Photovoltaic Array Performance." Solar Energy, Elsevier 80: 78-88.

[13] Villalva, M. G., Gazoli, J. R., and Filho, E. R. 2009. "Compreensive Approach to Modeling and Simulation of Photovoltaic Arrays." IEEE Trans. on Power Electronics, 1198-208.

[14] Wang, Y.-B., Wu, C.-S., Liao, H., and Xu, H.-H. 2008. "Steady-State Model and Power Flow Analysis of Grid Connected Photovoltaic Power System." In IEEE International Conference on Industrial Technology, Chengdu, 1-6.

[15] Junior Rangel, E. L. 2015. "Modelagem de Centrais Fotovoltaica no Problema de Fluxo de Potência Ótimo." Universidade Federal de Santa Catarina.

[16] Tsai, H.-L., Tu, C.-S., and Su, Y.-J. 2008. "Development of Generalized Photovoltaic Model Using MATLAB/Simulink.” In World Congress on Engineering and Computer Science, 1-6.

[17] Monticelli, A. J. 1983. Fluxo de Carga em Redes de Energia Elétrica. Edgard Blücher Ltda.

[18] Fang, S.-C., and Puthempura, S. 2003. Linear Optimization and Extensions. Prentice-Hall.

[19] IEEE Test Feeders. 2013, April. IEEE Test Feeders. [Online]. Available: http://ewh.ieee.org/soc/.

[20] Chiang, H., and Jean-Jumeau, R. Oct. 1990. "Optimal Network Reconfiguration in Distribution System. Part 1: A New Formulation and Solution Methodology." IEEE Trans. on Power Delivery 5: 1902-9. 